\title{
DIRICHLET FORMS ASSOCIATED WTTH HYPERCONTRACTIVE SEMIGROUPS
}

BY

JAMES G. HOOTON

\begin{abstract}
We exhibit a class of probability measures on $\mathbf{R}^{n}$ such that the associated Dirichlet form is represented by a selfadjoint operator $A$ and such that $e^{-\mu}$ is a hypercontractive semigroup of operators. The measures are of the form $d \mu=\Omega^{2} d x$ where $\Omega$ has classical first derivatives and $L^{p}$ second derivatives, $p$ determined by $n$.
\end{abstract}

1. Introduction. Given a probability measure $\mu$ on $\mathbf{R}^{n}$, we have an associated Dirichlet form

$$
h(u, v)=\int_{\mathbf{R}^{n}} \nabla u \cdot \overline{\nabla v} d \mu .
$$

For Gauss measure, $d \nu=(2 \pi)^{-n / 2} e^{-x^{2} / 2} d x$, it is well known that the selfadjoint operator $A u=-\Delta u+x \cdot \nabla u$ associated with the Dirichlet form generates a hypercontractive semigroup on the spaces $L^{p}\left(\mathbf{R}^{n}, d v\right), 1<p<\infty$ (see e.g. [7] or [11]).

L. Gross [4] developed a relationship between logarithmic Sobolev inequalities for a positive selfadjoint operator $A$ and hypercontractivity of the semigroup $e^{-\Lambda}$. J.-P. Eckmann [2] used this relationship to prove hypercontractivity for a class of measures of the form $d \mu=\Omega^{2} d x$ where $\Omega$ is strictly positive, spherically symmetric, and $C^{\infty}$ away from the origin.

Eckmann gave conditions on a potential $V$ which guarantee that $-\Delta+V$ is a positive selfadjoint operator on $L^{2}\left(\mathbf{R}^{n}, d x\right)$ which has a strictly positive eigenfunction $\Omega$ (taken to be normalized) corresponding to the eigenvalue 0 . For such a function $\Omega$, multiplication by $\Omega^{-1}$ is a unitary map from $L^{2}\left(\mathbf{R}^{n}, d x\right)$ to the probability space $L^{2}\left(\mathbf{R}^{n}, \Omega^{2} d x\right)$. He showed that $-\Delta+V$ corresponds via the map $\Omega^{-1}$ to a selfadjoint operator $A$ on $L^{2}\left(R^{n}, \Omega^{2} d x\right)$ associated with the Dirichlet form, and that $A$ generates a hypercontractive semigroup.

Instead of imposing conditions on potentials in order to produce measures, we have chosen to examine, in general, properties of a measure which imply hypercontractivity. The purpose of this paper is (i) to determine conditions on

Received by the editors June 26, 1978.

AMS (MOS) subject classifications (1970). Primary 47D05; Secondary 47F05, 47B25.

Key words and phrases. Dirichlet form, hypercontractive semigroup, logarithmic Sobolev inequality, $L^{P}$-contractive semigroup. 
a probability measure $\mu$ on $\mathbf{R}^{n}$ which guarantee existence of a selfadjoint Dirichlet operator associated with the Dirichlet form and (ii) to determine additional conditions which guarantee the hypercontractivity of $e^{-\mu}$.

In \$2, we fix a natural domain $\operatorname{Dom}(h)$ for the Dirichlet form $h$, and show that for measures of the form $d \mu=\Omega^{2} d x$ where $\Omega$ is a continuous nonnegative function with $L^{2}$ first derivatives, an associated operator exists on $\operatorname{Dom}(h)$, namely

$$
H u=-\Delta u-2 \frac{\nabla \Omega \cdot \nabla u}{\Omega} .
$$

If $H$ is essentially selfadjoint on $\operatorname{Dom}(h)$, then $\bar{H}$ is a positive selfadjoint operator whose behavior is determined by the behavior of $h$.

Suppose $d \mu=\Omega^{2} d x$ where $\Omega$ is $C^{1}$ with $L^{2}$ second derivatives. Under the unitary map $\Omega, H$ is transformed into the operator

$$
S \psi=-\Delta \psi+\frac{\Delta \Omega}{\Omega} \psi
$$

for $\psi \in \dot{\Omega} \operatorname{Dom}(h) \subset L^{2}\left(\mathbf{R}^{n}, d x\right)$. We have chosen $\operatorname{Dom}(h)$ so that $\Omega \operatorname{Dom}(h)$ $\supset C_{0}^{\infty}\left(\mathbf{R}^{n}\right)$, hence $S$ is a perturbation of $-\Delta$ on the domain $C_{0}^{\infty}\left(\mathbf{R}^{n}\right)$. Using standard results about perturbations of $-\Delta$, we can impose conditions on $\Delta \Omega / \Omega$ so that $S$ is essentially selfadjoint on $\Omega \operatorname{Dom}(h)$, in which case $H$ is essentially selfadjoint on $\operatorname{Dom}(h)$.

Given a positive selfadjoint operator $A$, the semigroup $e^{-\ell A}$ is automatically positivity preserving and $L^{p}$-contractive, as is shown in $\$ 3$. Theorem 4.5 generalizes Gross' Theorem 6 in [4] which indicates that if the operator $A$ satisfies a given logarithmic Sobolev inequality, then $e^{-4}$ is hypercontractive.

Finally, in $\$ 5$, we place additional conditions on the measures discussed in $\$ 2$, exhibiting a class of measures which satisfy the conditions of Theorem 4.5. We do not impose additional differentiability conditions on $\Omega$, thereby showing that the $C^{\infty}$ condition used by Eckmann [2] is more restrictive than necessary.

2. Essential selfadjointmess of the associated operator. We begin with standard definitions from the theory of distributions. We denote by $\mathscr{D}\left(R^{n}\right)=$ $C_{0}^{\infty}\left(\mathbf{R}^{n}\right)$ the set of infinitely differentiable functions with compact support in $\mathbf{R}^{n}$, and by $\mathscr{Q}^{\prime}\left(\mathbf{R}^{n}\right)$ the set of distributions on $\mathbf{R}^{n}$. A multi-index is a vector $\alpha=\left(\alpha_{1}, \ldots, \alpha_{n}\right)$ whose entries are nonnegative integers. For $f \in \Phi^{\prime}\left(R^{n}\right)$, we write

$$
D^{\alpha} f=\frac{\partial^{|\alpha|} f}{\partial x_{1}^{\alpha_{1}} \cdots \partial x_{n}^{\alpha_{n}}} \quad \text { where }|\alpha|=\sum_{i=1}^{n} \alpha_{i}
$$

We also write

$$
\frac{\partial f}{\partial x}=f_{x_{i}}, \quad \frac{\partial^{2} f}{\partial x_{i} \partial x_{j}}=f_{x_{i} x_{j}}
$$


when convenient. All derivatives are taken in the weak $\left(\Phi^{\prime}\left(\mathbf{R}^{n}\right)\right)$ sense. We note that for a sufficiently differential function $f$, the weak derivative $D^{\alpha} f$ is the classical derivative. The gradient of $f$, denoted $\nabla f$, is defined by $\nabla f=$ $\left(f_{x_{1}}, \ldots, f_{x_{n}}\right)$; the Laplacian, denoted $\Delta f$, is defined by

$$
\Delta f=\sum_{i=1}^{n} f_{x_{1} x_{j}} .
$$

For a measure $\mu$ on $\mathbf{R}^{n}, L^{p}\left(\mathbf{R}^{n}, d \mu\right)$ represents the standard $L^{p}$ space with respect to $\mu$. $L_{\text {loc }}^{p}\left(\mathbf{R}^{n}, d \mu\right)$ is the set of all functions $f$ such that $f \in L^{p}(K, d \mu)$ for all compact sets $K \subset \mathbf{R}^{n}$. Lebesgue measure on $\mathbf{R}^{n}$ is represented by $d x$.

Consider a probability measure $\mu$ on $\mathbf{R}^{n}$ and set $\mathcal{X}=L^{2}\left(\mathbf{R}^{n}, d \mu\right)$. For $u$, $v \in \mathcal{K}$, the inner product is written

$$
\langle u, v\rangle_{d \mu}=\int_{\mathbf{R}^{n}} u \bar{v} d \mu .
$$

Definition. We define the Dirichlet form $h$ by

$$
h(u, v)=\int_{\mathbf{R}^{n}} \nabla u \cdot \overline{\nabla v} d \mu
$$

with domain $\operatorname{Dom}(h)=\left\{u \in C^{1}\left(\mathbf{R}^{n}\right)\right.$ such that $D^{a} u$ is bounded for $|\alpha|<1$ and $D^{\alpha} u \in \mathcal{C}$ for $\left.|\alpha|=2\right\}$.

DEFINITION. We say that $h$ is represented by a Dirichlet operator $H$ if there exists on $\mathcal{X}$ an operator $H$ with $\operatorname{Dom}(H) \supset \operatorname{Dom}(h)$ such that

$$
h(u, v)=\langle H u, v\rangle_{d \mu}
$$

for all $u, v \in \operatorname{Dom}(h)$. We sometimes refer to $H$ as the operator associated with the Dirichlet form $h$.

We suppose now that $\mu$ is a measure on $\mathbf{R}^{n}$ such that $d \mu=\Omega^{2} d x$ where $\Omega$ satisfies

(i) $\Omega \in C\left(\mathbf{R}^{n}\right), \Omega>0$ on $\mathbf{R}^{n}$,

(ii) $D^{\alpha} \Omega \in L^{2}\left(\mathbf{R}^{n}, d x\right),|\alpha|<1$,

(iii) $\int_{\mathbf{R}^{n}} \Omega^{2} d x=1$.

For $u \in \operatorname{Dom}(h)$, we have $u_{x_{1}} \Omega^{2} \in C\left(\mathbf{R}^{n}\right) \cap L^{1}\left(\mathbf{R}^{n}, d x\right)$ and $\left(u_{x_{1}} \Omega^{2}\right)_{x_{1}}=u_{x_{1} x_{1}} \Omega^{2}$ $+2 u_{x_{1}} \Omega \Omega_{x_{i}} \in L^{1}\left(\mathbf{R}^{n}, d x\right)$ for $i=1, \ldots, n$. Hence for $v \in C^{1}\left(\mathbf{R}^{n}\right)$ such that $D^{\alpha} v$ is bounded for $|\alpha|<1$, it follows, by doing a standard integration by parts calculation using distribution theory, that

$$
\begin{aligned}
\int_{\mathbf{R}^{n}} \nabla u \cdot \overline{\nabla v} \Omega^{2} d x & =\int_{\mathbf{R}^{n}}\left(-(\Delta u) \Omega^{2}-2(\nabla \Omega \cdot \nabla u) \Omega\right) \bar{v} d x \\
& =\int_{\mathbf{R}^{n}}\left(-\Delta u-2 \frac{\nabla \Omega \cdot \nabla u}{\Omega}\right) \bar{v} \Omega^{2} d x
\end{aligned}
$$

where we adapt the convention that $\nabla \Omega(x) / \Omega=0$ if $\Omega(x)=0$. In particular, the Dirichlet form $h$ on $L^{2}\left(\mathbf{R}^{n}, d \mu\right)$ is represented by 


$$
H u=-\Delta u-2(\nabla \Omega \cdot \nabla u) / \Omega
$$

with $\operatorname{Dom}(H)$ taken to be $\operatorname{Dom}(h)$.

An essential part of the above integration by parts calculation consists in a product rule for distributions, namely for $f, g \in C\left(\mathbf{R}^{n}\right)$ such that $f_{x_{1}} \in$ $L^{p}\left(\mathbf{R}^{n}, d x\right), 1<p<\infty$, or $f_{x_{1}} \in L^{\infty}\left(\mathbf{R}^{n}\right) \cap C\left(\mathbf{R}^{n}\right)$ and $g_{x_{1}} \in L_{\text {loc }}^{1}\left(\mathbf{R}^{n}, d x\right)$, we have

$$
(f g)_{x_{i}}=f g_{x_{i}}+f_{x_{1}} g \text {. }
$$

We will encounter similar integration by parts and product rule calculations throughout this paper.

REMARK. Henceforth $h$ will represent the Dirichlet form for the measure considered and $H$ the associated operator on $\mathcal{H}=L^{2}\left(\mathbf{R}^{n}, d \mu\right)$. We set once and for all $\operatorname{Dom}(h)=\operatorname{Dom}(H)=\left\{u \in C^{1}\left(\mathbf{R}^{n}\right)\right.$ such that $D^{\alpha} u$ is bounded for $|\alpha|<1$ and $D^{\alpha} u \in \mathcal{H}$ for $\left.|\alpha|=2\right\}$.

REMARK. We will see shortly that our choice of $\operatorname{Dom}(h)$ is useful in proving essential selfadjointness of the associated operator $H$ for a wide class of measures. We have tried to restrict second derivatives as little as possible while still obtaining $H$ as a map from $\operatorname{Dom}(h)$ into $\mathscr{H}$. Gross [4] and Eckmann [2] used as domain for the Dirichlet form $\left\{u \in C^{2}\left(\mathbf{R}^{n}\right)\right.$ such that $D^{\alpha} u$ is bounded for $\left.|\alpha| \leqslant 2\right\}$. This domain is too restrictive for measures which are less smooth than those which they considered. It will become evident that the results of Gross and Eckmann hold on our larger domain.

REMARK. It does not seem as if $\mu$ can be much more singular and have a Dirichlet form represented in our sense by an operator. For example, if a measure on $\mathbf{R}^{1}$ is not absolutely continuous with respect to Lebesgue measure, then it can be shown that the Dirichlet form is not closable. An associated operator would necessarily be symmetric and hence could only represent a closable form. Or if $d \mu=\Omega^{2} d x$ where $\Omega$ is not continuous, then the domain $\operatorname{Dom}(H)$ must be adjusted to accommodate the discontinuities, a task which will not be undertaken in this paper.

In determining whether the operator $H$ is essentially selfadjoint, it will be advantageous to consider multiplication by $\Omega$ as inducing an isomorphism of the spaces $\mathcal{C}=L^{2}\left(\mathbf{R}^{n}, \Omega^{2} d x\right)$ with $L^{2}\left(\mathbf{R}^{n}, d x\right)$. For $\Omega$ suitably restricted, $S=\Omega H \Omega^{-1}$ is a well-known operator on $L^{2}\left(\mathbf{R}^{n}, d x\right)$. By appealing to the essential selfadjointness of $S$ and applying the isomorphism $\Omega$, we will show that $H$ is essentially selfadjoint.

LEMMA 2.1. Suppose $d \mu=\Omega^{2} d x$ where

(i) $\Omega \in C^{1}\left(\mathbf{R}^{n}\right), \Omega>0$ on $\mathbf{R}^{n}$,

(ii) $D^{\alpha} \Omega \in L^{2}\left(\mathbf{R}^{n}, d x\right)$ for $|\alpha|<2$,

(iii) $\int_{\mathbf{R}^{n}} \Omega^{2} d x=1$. 
Then for all $\psi \in \Omega \operatorname{Dom}(H)=\{\Omega u: u \in \operatorname{Dom}(H)\}$, we have $S \psi=-\Delta \psi+$ $V \psi$ where

$$
V= \begin{cases}\Delta \Omega / \Omega & \text { if } \Omega \neq 0, \\ 0 & \text { if } \Omega=0 .\end{cases}
$$

Proof. Suppose $\psi=\Omega u, u \in \operatorname{Dom}(H)$. Then $\psi_{x_{1}}=\Omega_{x_{1}} u+\Omega u_{x_{1}}$ and $\psi_{x_{1} x_{1}}=$ $\Omega_{x_{i} x_{i}} u+2 \Omega_{x_{i}} u_{x_{i}}+\Omega u_{x_{i} x_{i}} \cdot \Delta \psi$ is in $L^{2}\left(\mathbf{R}^{n}, d x\right)$ and we calculate

$$
S(\psi)=\Omega(H u)=-\Omega \sum_{i=1}^{n} u_{x_{1} x_{i}}-2 \sum_{i=1}^{n} u_{x_{i}} \Omega_{x_{i}}=-\Delta \psi+V \psi
$$

Hence the lemma follows.

$H$ is positive (hence symmetric) on its domain so that it certainly has a selfadjoint extension, namely its Friedrich's extension. If $H$ is essentially selfadjoint, i.e. if the operator closure of $H$ (denoted $\bar{H}$ ) is selfadjoint, then the behavior of the Dirichlet form on the natural domain $\operatorname{Dom}(h)$ uniquely determines the behavior of the selfadjoint extension.

REMARK. For the remainder of this paper we will assume that $\Omega(x)>0$ for all $x \in \mathbf{R}^{n}$. It should be mentioned that there are examples of measures with supports other than $\mathbf{R}^{n}$ such that the associated operator is essentially selfadjoint on $\operatorname{Dom}(h)$, but the proofs do not fit into the general theory which we are presenting here.

THEOREM 2.2. Suppose $d \mu=\Omega^{2} d x$ where

(i) $\Omega \in C^{1}\left(\mathbf{R}^{n}\right), \Omega(x)>0$ for all $x \in \mathbf{R}^{n}$,

(ii) $D^{\alpha} \Omega \in L^{2}\left(\mathbf{R}^{n}, d x\right),|\alpha|<2$,

(iii) $\int_{\mathbf{R}^{n}} \Omega^{2} d x=1$,

(iv) $V^{-}=-\min (0, V) \in L^{p}\left(\mathbf{R}^{n}, d x\right)+L^{\infty}\left(\mathbf{R}^{n}\right)$ for some $p>2, p>n / 2$.

Then $H$ is essentially selfadjoint on $\operatorname{Dom}(H)=\left\{u \in C^{1}\left(\mathbf{R}^{n}\right)\right.$ such that $D^{a_{u}}$ is bounded for $|\alpha|<1$ and $D a u \in \mathcal{C}$ for $|\alpha|=2\}$.

Proof. If we define $\operatorname{Dom}(S)=C_{0}^{\infty}\left(\mathbf{R}^{n}\right)$, then $S$ is essentially selfadjoint by a theorem of Kato [0] which states that if $V^{+}=\max (V, 0) \in L_{\text {loc }}^{2}\left(\mathbf{R}^{n}, d x\right)$ and $V^{-} \in L^{p}\left(\mathbf{R}^{n}, d x\right), p$ as above, then $-\Delta+V$ is essentially selfadjoint on $C_{0}^{\infty}\left(\mathbf{R}^{n}\right)$. We have $V^{+} \in L_{\text {loc }}^{2}\left(\mathbf{R}^{n}\right)$ since $\Omega$ is locally bounded away from zero.

LemMa 2.3. With $\operatorname{Dom}(S)=C_{0}^{\infty}\left(\mathbf{R}^{n}\right)$,

$$
\Omega^{-1} \operatorname{Dom}(S) \subset \operatorname{Dom}(H) \subset \Omega^{-1} \operatorname{Dom}(\bar{S})
$$

or equivalently,

$$
\operatorname{Dom}(S) \subset \Omega \operatorname{Dom}(H) \subset \operatorname{Dom}(\bar{S})
$$

where $\bar{S}$ represents the operator closure of $S$. 
PRoof. First inclusion. For $\psi \in C_{0}^{\infty}\left(\mathbf{R}^{n}\right), u=\Omega^{-1} \psi$, we have $u_{x_{1}}=$ $-\Omega^{-2} \Omega_{x_{i}} \psi+\Omega^{-1} \psi_{x_{i}}$ which is bounded and continuous. And $u_{x_{1} x_{i}}=$ $2 \Omega^{-3}\left(\Omega_{x_{i}}\right)^{2} \psi-\Omega^{-2} \Omega_{x_{i} x_{i}} \psi-2 \Omega^{-2} \Omega_{x_{i}} \psi_{x_{i}}+\Omega^{-1} \psi_{x_{i} x_{i}}$ which is in $\mathcal{H}$.

Second inclusion. For $u \in \operatorname{Dom}(H), \psi=\Omega u$, we showed in the proof of Lemma 2.1 that $\psi$ has continuous first derivatives and second derivatives in $L^{2}\left(\mathbf{R}^{n}, d x\right) . \psi$ is thus in the domain of the closed operator $-\Delta$. And $\psi$ is in the domain of the operator $V=V I$, where $I$ is the identity operator, since

$$
(\Delta \Omega / \Omega) \psi=(\Delta \Omega) u \in L^{2}\left(\mathbf{R}^{n}, d x\right) .
$$

Thus $\psi \in \operatorname{Dom}(-\Delta) \cap \operatorname{Dom}(V) \subset \operatorname{Dom}(\bar{S})$.

REMARK. Given Gross' and Eckmann's domain for the Dirichlet operator $H$, this lemma would not hold.

To complete the proof of the theorem, we note that since $\Omega$ is an isomorphism of Hilbert spaces, $H$ is essentially selfadjoint on $\Omega^{-1} \operatorname{Dom}(S)$. Since $H$ is symmetric on the larger subspace $\operatorname{Dom}(H)$, it is a standard result that $H$ is essentially selfadjoint on $\operatorname{Dom}(H)$.

REMARK. Condition (iii) is satisfied, for example, by any $\Omega$ such that $\Delta \Omega>0$ for $|x|>R$ and $\Delta \Omega \in L^{P}(\{x$ such that $|x|<R\}, d x)$. (iii) is a technical condition, used to insure the selfadjointness of $S$. Given a better perturbation theorem, condition (iii) might be weakened.

REMARK. A Dirichlet operator is positive, hence has an operator closure $\bar{H}$, a closed symmetric operator. In turn, $\bar{H}$ has a form closure $\hat{h}$. It is easy to show that $\hat{h}=\bar{h}$, where $\bar{h}$ is the closure of the Dirichlet form. In particular, if the Dirichlet form $h$ can be represented by an operator, then $h$ is closable.

Moreover, by the representation theorem for closed positive forms (see for example Theorem VI-(2.1) in [5]), $\bar{h}$ is represented by a selfadjoint operator $\hat{H}$. If $\bar{H}$ is itself selfadjoint, then it is easy to show that $\hat{H}=\bar{H}$.

REMARK. The proof of Theorem 2.2 relies on standard results concerning perturbations of $-\Delta$ on its natural domain $C_{0}^{\infty}\left(\mathbf{R}^{n}\right)$. For a more singular $\Omega, H$ no longer corresponds to a perturbation of $-\Delta$ on its natural domain. It can be shown on $R^{1}$ that the introduction of a simple jump into $\Omega^{\prime}$ does not affect the essential selfadjointness of $H$, but the proof is rather involved. As an example, however, it is not hard to show essential selfadjointness of the Dirichlet operator associated with the measure $d \mu=\Omega^{2} d x, \Omega(x)=e^{-|x|}$, on $\mathbf{R}^{1}$.

3. $L^{p}$-contractive semigroups. In this section, we see that a selfadjoint operator associated with the Dirichlet form on a probability space generates a positivity preserving, $L^{P}$-contractive semigroup. In $\$ 4$ we will discuss additional conditions on the measure so that the operator generates a hypercontractive semigroup.

A set of operators $\left\{T_{t}\right\}_{t \in[0, \infty)}$ on a Banach space $X$ is called a contraction 
semigroup if $T_{t+s}=T_{t} T_{s}$ and $\left\|T_{t}\right\|<1$ for $s, t \in[0, \infty)$, where $\|\cdot\|$ represents the operator norm. The infinitesimal generator $A$ of the semigroup is defined by

$$
A(x)=\operatorname{st}_{t \rightarrow 0}\left(\frac{T_{t}(x)-x}{t}\right)
$$

with domain consisting of all $x$ such that the limit exists. $A$ is a closed operator. We adopt the notation $T_{t}=e^{-\mu}$.

It is a standard result that a positive selfadjoint operator $A$ on a Hilbert space $\mathcal{K}$ is the generator of a (uniquely determined) contraction semigroup on $\mathcal{H}$.

We say that a contraction semigroup $\left\{e^{-\mu}\right\}$ on $L^{2}\left(\mathbf{R}^{n}, d \mu\right)$ is $L^{p}$-contractive if $\left\|e^{-t A} f\right\|_{p}<\|f\|_{p}$ for all $f \in L^{2}\left(\mathbf{R}^{n}, d \mu\right) \cap L^{p}\left(\mathbf{R}^{n}, d \mu\right), 1<p<\infty$. It is clear then that $\left\{e^{-i A}\right\}$ is a contraction semigroup on $L^{p}\left(\mathbf{R}^{n}, d \mu\right)$ for $p>2$ and that $\left\{e^{-t H}\right\}$ is a contraction semigroup on $L^{p}\left(\mathbf{R}^{n}, d \mu\right)$ for $p<2$, the closure taken in $L^{p}$. It is easy to show that the generator of the semigroup is $\left.A\right|_{L^{p}\left(\mathbf{R}^{n}, d \mu\right)}$ for $p>2$, and the closure of $A$ in $L^{p}\left(\mathbf{R}^{n}, d \mu\right)$ for $p<2$.

An operator $A$ on a space $L^{p}\left(\mathbf{R}^{n}, d \mu\right)$ is called positivity preserving if $A(f)$ is positive whenever $f$ is positive. A semigroup $e^{-t A}, t \in[0, \infty)$, is called positivity preserving if $e^{-\mu}$ is positivity preserving for each $t$.

Proposition 3.1. Suppose $d \mu=\Omega^{2} d x$ is a probability measure on $\mathbf{R}^{n}$ where $\Omega$ is a continuous, strictly positive function. If the Dirichlet form $h$ can be represented by an essentially selfadjoint operator $H$ with $\operatorname{Dom}(H)=\{u \in$ $C^{1}\left(\mathbf{R}^{n}\right)$ such that $D^{\alpha} u$ is bounded for $|\alpha|<1, D^{\alpha} u \in \mathcal{C}$ for $\left.|\alpha|=2\right\}$, then $\left\{e^{-t \bar{H}}\right\}_{t \in[0, \infty)}$ is a positivity preserving semigroup of operators.

Proof. Throughout the proof, we write $\langle\cdot, \cdot\rangle=\langle\cdot, \cdot\rangle_{d \mu}$ and $\bar{h}(u)=$ $\bar{h}(u, u)$.

The first Beurling-Deny criterion (see Beurling, Deny [1] or Theorem XIII.50 in [9]) gives several conditions on a positive selfadjoint operator $A$ on a Hilbert space $\mathcal{H}=L^{2}\left(\mathbf{R}^{n}, d \mu\right)$ which are equivalent to $e^{-\mu}$ being positivity preserving for all $t>0$. We show that $\bar{H}$ satisfies one of these conditions, namely: $e^{-\imath \bar{H}}$ is reality preserving and

$$
\bar{h}\left(u_{+}\right)+\bar{h}\left(u_{-}\right)<\bar{h}(u)
$$

for all real valued $u$ in the domain of the form $\bar{h}$ associated with $\bar{H}$, where $u_{+}(x)=\max (u(x), 0)$ and $u_{-}(x)=u_{+}(x)-u(x)$.

For convenience, we write $A=\bar{H}$. We let $Q(A)$ denote the form domain of $A$ and note that $Q(A)=\operatorname{Dom}(\bar{h}): \operatorname{Dom}(\bar{h})$ is the closure of $\operatorname{Dom}(h)$ with respect to the norm $\|u\|_{h}=(\|u\|+h(u))^{1 / 2}$. Equivalently, $Q(A)$ is the closure of $\operatorname{Dom}(H)=\operatorname{Dom}(h)$ with respect to the norm $\|u\|_{Q}=\left(\|u\|^{2}+\langle H u, u\rangle\right)^{1 / 2}$ $=\|u\|_{h}$. 
LEMMA 3.2. Given the conditions of Proposition 3.1, $e^{-M}$ is reality preserving for $t>0$.

Proof. For $u, v \in \operatorname{Dom}(H)$,

$$
\langle H \bar{u}, v\rangle=\int_{\mathbf{R}^{n}} \overline{\nabla u} \cdot \overline{\nabla v} d \mu=\overline{\langle H u, \bar{v}\rangle}=\langle\overline{H u}, v\rangle .
$$

Since conjugation is a continuous function, we have $A \bar{u}=\overline{A u}$ for all $u \in$ $\operatorname{Dom}(A)$. Next, for $v \in \mathcal{H}$ and $\lambda>0$, let $u=(A+\lambda)^{-1} v(\lambda \in \rho(A))$. Thus $v=(A+\lambda) u$ and $\bar{v}=(A+\lambda) \bar{u}$, so

$$
(A+\lambda)^{-1} \bar{v}=\overline{(A+\lambda)^{-1} v} \text {. }
$$

Finally, for $v \in \mathcal{K}, t>0$, we have

$$
e^{-u} v=\lim _{m \rightarrow \infty}\left(1-\frac{t}{m} A\right)^{-m} v .
$$

It follows from (2) that $e^{-\mu} \bar{v}=\overline{e^{-\mu} v}$. Hence $e^{-\mu}$ is reality preserving.

Continuation of PROOF OF Proposirion 3.1. The remainder of the proof consists of showing that $\bar{h}(|u|)<\bar{h}(u)$ for real valued $u \in Q(A)$, from which equation (1) will follow. To this end, it is instructive to characterize the elements of $Q(A)$.

Since $\Omega$ is continuous and strictly positive, $\mathcal{K} \subset L_{\text {loc }}^{1}\left(\mathbf{R}^{n}, d x\right)$. Thus $u \in$ $Q(A)$ has weak derivatives $u_{x_{1}}, \ldots, u_{x_{n}}$. We show that $u_{x_{1}} \in \mathcal{K}$ for $i=$ $1, \ldots, n$. Since $u \in Q(A)$, there exist $u_{k} \in \operatorname{Dom}(H)$ such that $\left\|u_{k}-u\right\| \rightarrow 0$ and $h\left(u_{k}-u_{j}\right) \rightarrow 0$ as $k, j \rightarrow \infty$. Hence

$$
\int_{\mathbf{R}^{n}}\left|\left(u_{k}\right)_{x_{i}}-\left(u_{j}\right)_{x_{i}}\right|^{2} d \mu \rightarrow 0 \text { for } i=1, \ldots, n
$$

so that $\left\{\left(u_{k}\right)_{x_{i}}\right\}$ is Cauchy in $\mathscr{C}$. Thus $\left\{\left(u_{k}\right)_{x_{i}}\right\} \rightarrow v_{i}$ in $\mathcal{X}$ and it is a standard distribution theory result that $v_{i}=u_{x_{i}}$ for $i=1, \ldots, n$.

In fact,

$$
h_{1}(u, v)=\int_{\mathbf{R}^{n}} \nabla u \cdot \overline{\nabla v} d \mu
$$

with $\operatorname{Dom}\left(h_{1}\right)=\left\{u \in \mathcal{H}\right.$ such that $\left.u_{x_{1}} \in \mathcal{H}, i=1, \ldots, n\right\}$ is a closed positive form and $h_{1} \supset h$. By the representation theorem for closed positive forms, there exists a selfadjoint operator $A_{1}$ which represents $h_{1}$. Since $A_{1} \supset A$, we have $A_{1}=A$ and thus $h_{1}=\bar{h}$. Hence $Q(A)=\{u \in \mathcal{X}$ such that $\left.u_{x_{1}} \in \mathcal{K}, i=1, \ldots, n\right\}$ and for $u \in Q(A)$,

$$
\bar{h}(u)=\int_{\mathbf{R}^{n}}|\nabla u|^{2} d \mu .
$$

Next, for real valued $u \in Q(A)$, we approximate $|u|$ by elements $f_{n}(u) \in$ $Q(A)$ such that $\bar{h}\left(f_{n}(u)\right)<\bar{h}(u)$. This will lead us to the desired result, $\bar{h}(|u|)<\bar{h}(u)$. 
Consider on $\mathbf{R}$

and finally

$$
\begin{aligned}
& \eta(x)= \begin{cases}e^{1 / x} & \text { for } x<0 \\
0 & \text { for } x>0\end{cases} \\
& g(x)=\eta\left(x^{2}-1\right)
\end{aligned}
$$

$$
\varphi(x)=g(x) / \int_{\mathbf{R}} g(x) d x .
$$

Then $\varphi(x)$ is an approximate identity on $\mathbf{R}$ which is symmetric with respect to the origin.

We let $f(x)=|x|$ on $\mathbf{R}$ and $f_{m}(x)=\varphi_{m} * f(x)=\int_{\mathbf{R}^{n}} \varphi_{m}(x-y) f(y) d y$ where $\varphi_{m}(x)=m \varphi(m x), m \in \mathbf{Z}^{+}$, and * represents convolution, defined as shown. The following properties are easily verified (see Figure 1).

(i) $\left|f_{m}(x)\right|<|x|+1 / m,\left|f_{m}^{\prime}\right|<1, f_{m}^{\prime \prime}$ is bounded for $m \in \mathbf{Z}^{+}$.

(ii) $f_{m} \rightarrow f$ uniformly on $\mathbf{R}$; in fact $f_{m}(x)=|x|$ for $|x|>1 / m$.

(iii) $\left|f_{m+1}^{\prime}(x)\right| \geqslant\left|f_{m}^{\prime}(x)\right|$ for $x \in \mathbf{R}, m \in \mathbf{Z}^{+}$.
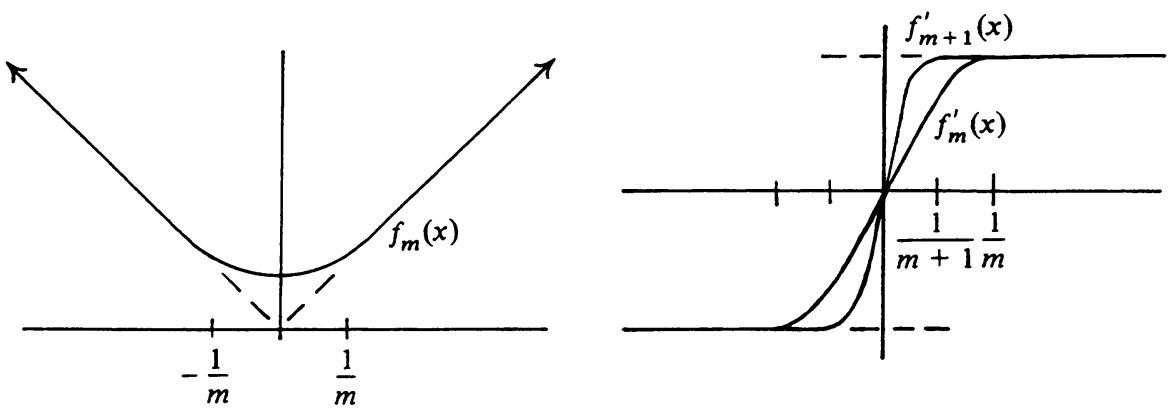

FiguRe 1

LEMMA 3.3. For $u \in Q(A)$, $u$ real valued, and $m \in \mathbf{Z}^{+}$, we have $f_{m}(u) \in$ $Q(A)$ and

$$
\bar{h}\left(f_{m}(u)\right)=\int_{\mathbf{R}^{n}}\left(f_{m}^{\prime}(u)\right)^{2}(\nabla u)^{2} d \mu .
$$

Proof. Suppose $u \in \operatorname{Dom}(H), u$ real valued. Then $f_{m}(u)$ is continuous and $\left|f_{m}(u)\right|<|u|+1 / m$. For $i=1, \ldots, n,\left(f_{m}(u)\right)_{x_{1}}=f_{m}^{\prime}(u) u_{x_{1}}$ is continuous and bounded since $\left|f_{m}^{\prime}\right|<1$. Also, $\left(f_{m}(u)\right)_{x_{1} x_{i}}=f_{m}^{\prime \prime}(u)\left(u_{x_{1}}\right)^{2}+f_{m}^{\prime}(u) u_{x_{1} x_{i}}$ is in $\mathcal{X}$ since $f_{m}^{\prime \prime}$ is bounded. Hence $f_{m}(u)$ is in $\operatorname{Dom}(H)$.

For $u \in Q(A), u$ real valued, $f_{m}(u)$ is in $\mathcal{X}$ since $\left|f_{m}(u)\right|<|u|+1 / m$. We show now that for $i=1, \ldots, n,\left(f_{m}(u)\right)_{x_{1}}=f_{m}^{\prime}(u) u_{x_{1}}$, which is in $\mathcal{H}$ since $\left|f_{m}^{\prime}\right|<1$. There exist $u_{k} \in \operatorname{Dom}(H)$ such that $\left\|u_{k}-u\right\| \rightarrow 0$ and $\|\left(u_{k}\right)_{x_{1}}-$ $u_{x_{i}} \| \rightarrow 0$ as $k \rightarrow \infty, i=1, \ldots, n$. We may, and do, assume that $u_{k}$ is real valued for $k \in \mathbf{Z}^{+}$. $\left\|f_{m}\left(u_{k}\right)-f_{m}(u)\right\|^{2}<\left\|u_{k}-u\right\|^{2}$ since $\left|f_{m}^{\prime}\right|<1$ so that 
$f_{m}\left(u_{k}\right) \rightarrow f_{m}(u)$ in $\mathcal{H}$. Also,

$$
\begin{aligned}
\int_{\mathbf{R}^{n}}\left|\left(f_{m}\left(u_{k}\right)\right)_{x_{i}}-f_{m}^{\prime}(u) u_{x_{i}}\right| d \mu \\
\quad<\int_{\mathbf{R}^{n}}\left|f_{m}^{\prime}\left(u_{k}\right)\right|\left|\left(u_{k}\right)_{x_{i}}-u_{x_{i}}\right| d \mu+\int_{\mathbf{R}^{n}}\left|f_{m}^{\prime}\left(u_{k}\right)-f_{m}^{\prime}(u)\right|\left|u_{x_{i}}\right| d \mu \\
\quad<\left(\int_{\mathbf{R}^{n}}\left|\left(u_{k}\right)_{x_{i}}-u_{x_{i}}\right|^{2} d \mu\right)^{1 / 2}+M\left(\int_{\mathbf{R}^{n}}\left|u_{k}-u\right|^{2} d \mu\right)^{1 / 2}\left(\int_{\mathbf{R}^{n}}\left|u_{x_{i}}\right|^{2} d \mu\right)^{1 / 2} \\
\quad \rightarrow 0
\end{aligned}
$$

as $k \rightarrow \infty$ where $M$ is a bound for $f_{m}^{\prime \prime}$. Hence we have $\left(f_{m}(u)\right)_{x_{1}}=f_{m}^{\prime}(u) u_{x_{1}}$ since $f_{m}\left(u_{k}\right) \rightarrow f_{m}(u)$ and $\left(f_{m}\left(u_{k}\right)\right)_{x_{i}} \rightarrow f_{m}^{\prime}(u) u_{x_{1}}$ in $L_{\text {loc }}^{1}\left(\mathbf{R}^{n}, d x\right)$. Thus $f_{m}(u) \in$ $Q(A)$ and the lemma follows.

LEMMA 3.4. If $u \in Q(A)$, $u$ real valued, then $|u| \in Q(A)$ and $\bar{h}(|u|)<\bar{h}(u)$.

Proof. Fix $u \in Q(A), u$ real valued, and define $\xi(m)=\bar{h}\left(f_{m}(u)\right)$ for $m \in \mathbf{Z}^{+}$. Since $\left|f_{m}^{\prime}\right|<1, \xi(m)<\bar{h}(u)$, and since $\left(f_{m+1}\right)^{\prime}(u)>f_{m}^{\prime}(u), \xi(m+1)$ $>\xi(m)$ for $m \in \mathbf{Z}^{+}$. Hence $\{\xi(m)\}$ is an increasing bounded sequence which has a limit $L<\bar{h}(u)$.

We have $\left\|f_{m}(u)-|u|\right\| \rightarrow 0$ since $f_{m} \rightarrow|\cdot|$ uniformly. It suffices to show $\bar{h}\left(f_{m}(u)-f_{k}(u)\right) \rightarrow 0$, which implies that $|u| \in Q(A)$ and $\bar{h}(|u|)=L$.

If $u(x)>0$ and $m>k$, then $f_{m}^{\prime}(u)>f_{k}^{\prime}(u)>0,2\left(f_{k}^{\prime}(u)\right)^{2}-2 f_{m}^{\prime}(u) f_{k}^{\prime}(u)<$ 0 , and finally,

$$
\left(f_{k}^{\prime}(u)-f_{m}^{\prime}(u)\right)^{2}<\left(f_{m}^{\prime}(u)\right)^{2}-\left(f_{k}^{\prime}(u)\right)^{2} .
$$

If $u(x)<0$ and $m>k$, then $f_{m}^{\prime}(u)<f_{k}^{\prime}(u)<0$ and similarly (3) holds. Hence for $m, k \in \mathbf{Z}^{+}$,

$$
\left(f_{k}^{\prime}(u)-f_{m}^{\prime}(u)\right)^{2}<\left|\left(f_{m}^{\prime}(u)\right)^{2}-\left(f_{k}^{\prime}(u)\right)^{2}\right| .
$$

But now

$$
\begin{aligned}
\bar{h}\left(f_{k}(u)-f_{m}(u)\right) & =\int_{\mathbf{R}^{n}}|\nabla u|^{2}\left(f_{k}^{\prime}(u)-f_{m}^{\prime}(u)\right)^{2} d \mu \\
& <\int_{\mathbf{R}^{n}}|\nabla u|^{2}\left|\left(f_{m}^{\prime}(u)\right)^{2}-\left(f_{k}^{\prime}(u)\right)^{2}\right| d \mu \rightarrow 0 \text { as } m, k \rightarrow \infty
\end{aligned}
$$

since $\xi(m)$ is a Cauchy sequence. Hence the lemma is proved.

Conclusion of proof of Proposition 3.1. Finally, for $u \in Q(A), u$ real valued, $u_{+}=(|u| / 2)+u / 2$ and $u_{-}=(|u| / 2)-u / 2$ are in $Q(A)$ and

$$
\begin{aligned}
\bar{h}\left(u_{+}\right)+\bar{h}\left(u_{-}\right)= & \frac{1}{4} \bar{h}(|u|)+\frac{1}{2} \bar{h}(|u|, u)+\frac{1}{4} \bar{h}(u) \\
& +\frac{1}{4} \bar{h}(|u|)-\frac{1}{2} \bar{h}(|u|, u)+\frac{1}{4} h(u) \\
< & \frac{1}{2} \bar{h}(|u|)+\frac{1}{2} \bar{h}(u)<\bar{h}(u) .
\end{aligned}
$$

Hence the proof is complete. 
Theorem 3.5. Given the condition of Proposition 3.1, $\left\{e^{-t \bar{H}}\right\}$ is an $L^{P}$-contractive semigroup which is strongly continuous for $1<p<\infty$.

Proof. We note that since $\bar{H}(1)=0$, then $e^{-t \bar{H}}(1)=1$. The theorem now follows from Theorem X.55 in [8] which states that if $A$ is a positive selfadjoint operator on $L^{2}\left(\mathbf{R}^{n}, d \mu\right), \mu$ a probability measure, and if $e^{-\mu}$ is positivity preserving with $e^{-M A}(1)=1$, then $e^{-t A}$ is $L^{P}$-contractive and, moreover, the semigroup is strongly continuous for $1<p<\infty$.

4. Sobolev generators and hypercontractivity. Suppose that $A$ is a positive selfadjoint operator on $L^{2}\left(\mathbf{R}^{n}, d \mu\right)$ where $\mu$ is a probability measure. We say that $e^{-\mu}$ is a hypercontractive semigroup on the spaces $L^{P}\left(\mathbf{R}^{n}, d \mu\right), 1<p<$ $\infty$, if $e^{-t A}$ is an $L^{P}$-contractive semigroup and if there exist constants $b>2$, $t_{b}>0$ and $C$ such that

$$
\left\|e^{-t_{b} A}\right\|_{2, b}<C
$$

where $\|\cdot\|_{p, q}$ represents the norm of an operator from $L^{p}\left(\mathbf{R}^{n}, d \mu\right)$ to $L^{q}\left(\mathbf{R}^{n}, d \mu\right)$.

We note that if $e^{-\mu}$ is a hypercontractive semigroup, then for $p, q \in$ $(1, \infty)$, there exist constants $t_{p q}>0$ and $C_{p q}$ such that if $t>t_{p q}$, then

$$
\left\|e^{-\mu}\right\|_{p, q}<C_{p q}
$$

This is shown, for example, in [8].

There is a useful relationship between hypercontractivity and logarithmic Sobolev inequalities. We begin with definitions, taken from [4].

For $u$ complex valued and $p \in(1, \infty)$, we define $u_{p}=(\operatorname{sgn} u)|u|^{p-1}$,

$$
\operatorname{sgn} u= \begin{cases}u /|u| & \text { if } u \neq 0, \\ 0 & \text { if } u=0 .\end{cases}
$$

For a probability space $\left(\mathrm{R}^{n}, \mu\right)$ and $p \in(1, \infty)$, we call a closed operator $A$ on $L^{p}\left(\mathbf{R}^{n}, d \mu\right)$ a Sobolev generator of index $p$ if it is the generator of a strongly continuous semigroup $e^{-\mu}$ on $L^{p}\left(\mathbf{R}^{n}, d \mu\right)$ and if for some real constants $c>0$ and $\gamma$ we have

$$
\int_{\mathbf{R}^{n}}|u|^{p} \ln |u| d \mu \leqslant c \operatorname{Re}\left\langle(A+\gamma) u, u_{p}\right\rangle+\|u\|_{p}^{p} \ln \|u\|_{p}
$$

for all $u \in \operatorname{Dom}(A)$, where

$$
\langle u, v\rangle=\int_{\mathbf{R}^{n}} u \bar{v} d \mu \text { and }\|u\|_{p}=\left(\int_{\mathbf{R}^{n}}|u|^{p} d \mu\right)^{1 / p} .
$$

We say that $c$ is the Sobolev coefficient and $\gamma$ is the local norm for $A$.

Suppose $1<a<b<\infty, r \in(a, b)$, and that $e^{-\mu}$ is a strongly continuous semigroup on $L^{r}\left(\mathbf{R}^{n}, d \mu\right)$ whose restriction to $L^{p}$ if $p>r$ or closure in $L^{p}$ if $p<r$ is strongly continuous in $L^{p}$ for all $p \in(a, b)$. We say that $A$ is a Sobolev generator on $(a, b)$ if there are continuous functions $c(\cdot)>0$ and 
$\gamma(\cdot)$ on $(a, b)$ such that for all $p \in(a, b)$, the generator in $L^{p}$ of the semigroup $e^{-\mu}$ is a Sobolev generator of index $p$ with Sobolev coefficient $c(p)$ and local norm $\gamma(p)$.

THEOREM 4.1. Suppose $\mu$ is a probability measure on $\mathbf{R}^{n}$. Suppose the Dirichlet form $h$ can be represented by an essentially selfadjoint operator $H$ with $\operatorname{Dom}(h)=\operatorname{Dom}(H)=\left\{u \in C^{1}\left(\mathbf{R}^{n}\right)\right.$ such that $D^{\alpha} u$ is bounded for $|\alpha|<1$ and $D^{\alpha} u \in \mathcal{C}=L^{2}\left(\mathbf{R}^{n}, d \mu\right)$ for $\left.|\alpha|=2\right\}$. If $\left\{e^{-t \bar{H}}\right\}_{t \in[0, \infty)}$ is a positivity preserving semigroup and if there exist constants $c>0$ and $\delta$ such that

$$
\int_{\mathbf{R}^{n}}|u|^{2} \ln |u| d \mu<c \int_{\mathbf{R}^{n}}|\nabla u|^{2} d \mu+\|u\|_{2}^{2} \ln \|u\|_{2}+\delta\|u\|_{2}^{2}
$$

for all $u \in \operatorname{Dom}(H)$, then $\bar{H}$ is a Sobolev generator on $[2, \infty)$.

REMARK. As in Theorem 3.5, it follows immediately that $\left\{e^{-\imath \bar{H}}\right\}$ is an $L^{P}$-contractive semigroup which is strongly continuous for $1<p<\infty$.

The proof proceeds as a sequence of lemmas. We set $\mathscr{D}_{1}=\left\{u \in C^{1}\left(\mathbf{R}^{n}\right)\right.$ such that $D^{\alpha} u$ is bounded for $|\alpha|<1$.

LEMMA 4.2. For $u \in \mathscr{D}_{1}, u$ real valued, and $p>2$, we have $|u|^{p / 2}, u_{p} \in \mathscr{D}_{1}$ and

$$
\int_{\mathbf{R}^{n}}\left(\nabla\left(|u|^{p / 2}\right)\right)^{2} d \mu=\frac{(p / 2)^{2}}{p-1} \int_{\mathbf{R}^{n}} \nabla u \cdot \nabla u_{p} d \mu .
$$

Proof. For $u \in \mathfrak{D}_{1}, u$ real valued, $p>2$, we have

$$
|u|^{p / 2}= \begin{cases}u^{p / 2} & \text { when } u(x)>0 \\ (-u)^{p / 2} & \text { when } u(x)<0\end{cases}
$$

and

$$
\left(|u|^{p / 2}\right)_{x_{i}}= \begin{cases}(p / 2) u^{(p-2) / 2} u_{x_{i}} & \text { when } u(x)>0, \\ -(p / 2)(-u)^{(p-2) / 2} u_{x_{i}} & \text { when } u(x)<0,\end{cases}
$$

for $i=1, \ldots, n$. Setting $\left(|u|^{p / 2}\right)_{x_{1}}=0$ when $u(x)=0$ and using a straightforward mean value argument, we see that $|u|^{p / 2} \in \mathfrak{D}_{1}$.

Similarly,

$$
u_{p}= \begin{cases}u^{p-1} & \text { when } u(x)>0 \\ -(-u)^{p-1} & \text { when } u(x)<0\end{cases}
$$

and

$$
\left(u_{p}\right)_{x_{i}}= \begin{cases}(p-1) u^{p-2} u_{x_{1}} & \text { when } u(x)>0, \\ (p-1)(-u)^{p-2} u_{x_{1}} & \text { when } u(x)<0 .\end{cases}
$$

Setting $\left(u_{p}\right)_{x_{1}}=0$ when $u(x)=0$, we see that $u_{p} \in \mathfrak{D}_{1}$ for $p>2$. 
Finally,

$$
\begin{aligned}
\left(\nabla\left(|u|^{p / 2}\right)\right)^{2} & = \begin{cases}\sum_{i=1}^{n}\left(\frac{p}{2}\right)^{2} u^{p-2}\left(u_{x_{i}}\right)^{2}, & u(x)>0, \\
\sum_{i=1}^{n}\left(\frac{p}{2}\right)^{2}(-u)^{p-2}\left(u_{x_{i}}\right)^{2}, & u(x)<0, \\
0, \quad u(x)=0,\end{cases} \\
& =\frac{(p / 2)^{2}}{p-1}\left(\nabla u \cdot \nabla u_{p}\right) .
\end{aligned}
$$

The lemma follows.

LEMMA 4.3. For $u \in \operatorname{Dom}(H)$, $u$ real valued, and $p>2$, we have

$$
\begin{aligned}
\int_{\mathbf{R}^{n}}|u|^{p} \ln |u| d \mu & <\frac{c}{2} \frac{p}{p-1} \int_{\mathbf{R}^{n}} \nabla u \cdot \nabla\left(u_{p}\right) d \mu+\|u\|_{p}^{p} \ln \|u\|_{p}+\frac{2 \delta}{p}\|u\|_{p}^{p} \\
& =\frac{c}{2} \frac{p}{p-1}\left\langle H u, u_{p}\right\rangle+\|u\|_{p}^{p} \ln \|u\|_{p}+\frac{2 \delta}{p}\|u\|_{p}^{p} .
\end{aligned}
$$

REMARK. Note that for $u$ real valued, equation (6) is the same as (4) with Sobolev coefficient $(c / 2)(p /(p-1))$ and local norm $4 \delta(p-1) / c p^{2}$.

Proof. The proof that

$$
\int_{\mathbf{R}^{n}} \nabla u \cdot \nabla\left(u_{p}\right) d \mu=\left\langle H u, u_{p}\right\rangle
$$

is a straightforward integration by parts calculation similar to the one described in \$2.

It is easy to show that $\mathscr{D}_{1} \subset Q(\bar{H})$. So for $u \in \mathscr{D}_{1}$, there exists $\left\{u_{k}\right\} \subset$ $\operatorname{Dom}(H)$ such that for $i=1, \ldots, n,\left\|u_{k}-u\right\|_{2} \rightarrow 0$ and $\left\|\left(u_{k}\right)_{x_{i}}-u_{x_{1}}\right\| \rightarrow 0$ as $k \rightarrow \infty$. Setting

$$
\begin{gathered}
a_{k}=c \int_{\mathbf{R}^{n}}\left|\nabla u_{k}\right|^{2} d \mu+\left\|u_{k}\right\|_{2}^{2} \ln \left\|u_{k}\right\|_{2}+\delta\left\|u_{k}\right\|_{2}^{2}, \\
a=c \int_{\mathbf{R}^{n}}|\nabla u|^{2} d \mu+\|u\|_{2}^{2} \ln \|u\|_{2}+\delta\|u\|_{2}^{2}
\end{gathered}
$$

we see that $a_{k} \rightarrow a$ as $k \rightarrow \infty$ since $x^{2} \ln x$ is continuous on $[0, \infty$ ) (here $x^{2} \ln x$ is defined to be 0 if $x=0$ ).

We set

$$
b_{k}=\int_{\mathbf{R}^{n}}\left|u_{k}\right| \ln \left|u_{k}\right| d \mu
$$

Since (5) holds for $u_{k}$ and since $x \ln x>-1$ for $x \in[0, \infty)$, it follows that $-1<b_{k}<a_{k}$ for $k \in Z^{+}$. Hence $\left\{b_{k}\right\}$ has a subsequence converging to $b$ where $-1<b<a$. We assume without loss of generality that the entire sequence converges to $b$. 
Since $u_{k} \rightarrow u$ in $\mathcal{H}$, some subsequence converges to $u$ almost everywhere. We assume that the sequence converges almost everywhere to $u$. Using Fatou's lemma for $\left|u_{k}\right| \ln \left|u_{k}\right|+1>0$, we have

$$
\begin{aligned}
\int_{\mathbf{R}^{n}}|u|^{2} \ln |u| d \mu+1 & <\lim _{k \rightarrow \infty} \int_{\mathbb{R}^{n}}\left(\left|u_{k}\right|^{2} \ln \left|u_{k}\right|+1\right) d \mu \\
& =b+1<a+1 .
\end{aligned}
$$

Hence for $u \in \mathfrak{D}_{1}$, (5) holds.

For $u \in \operatorname{Dom}(H), u$ real valued, $p>2$ we have $|u|^{p / 2} \in \mathfrak{D}_{1}$. Substituting $|u|^{p / 2}$ into (5), then (6) follows.

Lemma 4.4. For $p>2$, we define $\operatorname{Dom}_{p}(H)=\{u \in \operatorname{Dom}(H)$ such that $\left.H u \in L^{p}\left(\mathbf{R}^{n}, d \mu\right)\right\}$. $H$ is closable on $\operatorname{Dom}_{p}(H)$ as an operator on $L^{p}\left(\mathbf{R}^{n}, d \mu\right)$. For $p>2$, we denote by $\bar{H}_{p}$ the $p$ closure of $H$. Then (6) holds for all real valued $u \in \operatorname{Dom}\left(\bar{H}_{p}\right), p>2$.

Proof. Suppose $\left\{u_{k}\right\} \subset \operatorname{Dom}_{p}(H), p>2$, and $u_{k} \rightarrow 0, H u_{k} \rightarrow v$ in $L^{p}\left(\mathbf{R}^{n}, d \mu\right)$. Since $L^{p}\left(\mathbf{R}^{n}, d \mu\right)$ is continuously imbedded in $\mathcal{H C}, u_{k} \rightarrow 0$ and $H u_{k} \rightarrow v$ in $\mathcal{H}$. But $H$ is closable on $\mathcal{K}$, so $v=0$. Hence $H$ is closable on $\operatorname{Dom}_{p}(H)$ as an operator on $L^{p}\left(\mathbf{R}^{n}, d \mu\right)$.

Suppose $u \in \operatorname{Dom}\left(\bar{H}_{p}\right), p>2$, and $u$ is real valued. There exist $u_{k}$ real valued in $\operatorname{Dom}_{p}(H)$ such that $\left\|u_{k}-u\right\|_{p} \rightarrow 0$ and $\left\|H u_{k}-\bar{H}_{p} u\right\|_{p} \rightarrow 0$. We set $w=\bar{H}_{p} u$.

For $q=p /(p-1), v \rightarrow v_{p}$ is a homeomorphism from $L^{p}\left(\mathbf{R}^{n}, d \mu\right)$ to $L^{q}\left(\mathbf{R}^{n}, d \mu\right)$. Thus

$$
\begin{aligned}
\mid\left\langle H u_{k},\left(u_{k}\right)_{p}\right\rangle & -\left\langle w, u_{p}\right\rangle \mid \\
& <\left|\left\langle H u_{k},\left(u_{k}\right)_{p}\right\rangle-\left\langle H u_{k}, u_{p}\right\rangle\right|+\left|\left\langle H u_{k}, u_{p}\right\rangle-\left\langle w, u_{p}\right\rangle\right| \\
& <\left\|H u_{k}\right\|_{p}\left\|\left(u_{k}\right)_{p}-u_{p}\right\|_{q}+\left\|H u_{k}-w\right\|_{p}\left\|u_{p}\right\|_{q} \rightarrow 0 \text { as } k \rightarrow \infty .
\end{aligned}
$$

Hence

$$
\begin{aligned}
\frac{c}{2} \frac{p}{p-1} & \left\langle H u_{k},\left(u_{k}\right)_{p}\right\rangle+\left\|u_{k}\right\|_{p}^{p} \ln \left\|u_{k}\right\|_{p}+\frac{2 \delta}{p}\left\|u_{k}\right\|_{p}^{p} \\
& \rightarrow \frac{c}{2} \frac{p}{p-1}\left\langle\bar{H}_{p} u, u_{p}\right\rangle+\|u\|_{p}^{p} \ln \|u\|_{p}+\frac{2 \delta}{p}\|u\|_{p}^{p} \text { as } k \rightarrow \infty .
\end{aligned}
$$

The lemma follows similarly as in the proof of Lemma 4.3.

Proof of Theorem 4.1. Corollary 2.1 in [4] states that if $\bar{H}$ is a Sobolev generator on $(a, b)$ with the exception that $(6)$ holds only for nonnegative $u \in \operatorname{Dom}\left(\bar{H}_{p}\right), p \in(a, b)$, and if $e^{-t \bar{H}}$ is positivity preserving, then $\bar{H}$ is a Sobolev generator on $(a, b)$ and (6) holds for all $u \in \operatorname{Dom}\left(\bar{H}_{p}\right)$. Hence the theorem follows.

Theorem 1 in [4] states the following: let $A$ be a Sobolev generator on $(a, b)$ with Sobolev coefficient function $c(\cdot)$ and local norm function $\gamma(\cdot)$. For each 
$q \in(a, b)$ let $p(t, q)$ be the solution of the initial value problem

$$
c(p) \frac{d p}{d t}=p, \quad p(0, q)=q, \quad t>0
$$

and let

$$
M(t, q)=\int_{0}^{t} \gamma(p(s, q)) d s .
$$

$p(t, q)$ and $M(t, q)$ are both defined as long as $p(t, q)<b$. Then

$$
\left\|e^{-u}\right\|_{q, p(i, q)}<e^{M(t, q)} \text {. }
$$

We use this theorem, which can easily be extended to apply to a Sobolev generator on an interval of the form $[a, b)$, to prove the following theorem on hypercontractivity.

TheOREM 4.5. Suppose $d \mu=\Omega^{2} d x$ is a probability measure on $\mathbf{R}^{n}$ where $\Omega$ is a strictly positive function. Suppose the Dirichlet form $h$ can be represented by an essentially selfadjoint operator $H$ with $\operatorname{Dom}(h)=\operatorname{Dom}(H)=\left\{u \in C^{1}\left(\mathbf{R}^{n}\right)\right.$ such that $D^{\alpha} u$ is bounded for $|\alpha|<1$ and $D^{\alpha} u \in \mathcal{C}=L^{2}\left(\mathbf{R}^{n}, d \mu\right)$ for $\left.|\alpha|=2\right\}$. If there exist constants $c>0$ and $\delta$ such that

$$
\int_{\mathbf{R}^{n}}|u|^{2} \ln |u| d \mu<c \int_{\mathbf{R}^{n}}|\nabla u|^{2} d \mu+\|u\|_{2}^{2} \ln \|u\|_{2}+\delta\|u\|_{2}^{2}
$$

for all $u \in \operatorname{Dom}(H)$, then $\bar{H}$ generates a hypercontractive semigroup on the spaces $L^{P}\left(\mathbf{R}^{n}, d \mu\right), 1<p<\infty$.

Proof. By Theorem 3.5, $\left\{e^{-\imath \bar{H}}\right\}$ is an $L^{P}$-contractive semigroup. Consider Theorem 1 in [4] with

$$
c(p)=\frac{c}{2} \frac{p}{p-1}, \quad \gamma(p)=\frac{4 \delta(p-1)}{c p^{2}} .
$$

The solution of

$$
c(p) \frac{d p}{d t}=p, \quad p(0)=2,
$$

increases without bound as $t \rightarrow \infty$. In particular, there exists $t_{1}$ such that $p\left(t_{1}\right)=4$. Also,

$$
M\left(t_{1}, 2\right)=\int_{0}^{2} \gamma(p(s)) d s<\infty
$$

so that

$$
\left\|e^{-t_{1} \bar{H}}\right\|_{2,4}<e^{M\left(t_{1}, 2\right)}<\infty .
$$

COROLLARY 4.6. Given the conditions of Theorem 3.5, $\bar{H}$ is a Sobolev generator on $(1, \infty)$. 
Proof. It is shown in [4] that the generator of a hypercontractive semigroup is a Sobolev generator on $(1, \infty)$.

REMARK. The results of this section generalize the domain requirements of Theorem 6 in [4]. An examination of the proofs of Theorems 4.1 and 4.5 will reveal that the theorems are valid on the more restrictive domain $\operatorname{Dom}(h)=$ $\operatorname{Dom}(H)=\left\{u \in C^{2}\left(\mathbf{R}^{n}\right)\right.$ such that $D^{\alpha} u$ is bounded for $\left.|\alpha|<2\right\}$ used in [4].

5. Logarithmic Sobolev inequalities. We develop in this section conditions on a probability measure so that the conditions of Theorem 4.5 are satisfied, in which case a hypercontractive semigroup is generated.

We define $\Re$ to be the set of all measures $\mu$ on $\mathbf{R}^{n}$ such that $d \mu=\Omega^{2} d x$ where $\Omega$ satisfies

(i) $\Omega \in C^{1}\left(\mathbf{R}^{n}\right), \Omega(x)>0$ for all $x \in \mathbf{R}^{n}$,

(ii) $D^{\alpha} \Omega \in L^{2}\left(\mathbf{R}^{n}, d x\right)$ for $|\alpha|<2$,

(iii) $\int_{\mathbf{R}^{n}} \Omega^{2} d x=1$,

(iv) $V^{-} \in L^{p}\left(\mathbf{R}^{n}, d x\right)$ where $V=\Delta \Omega / \Omega$ for some $p>2, p>n / 2$,

(v) $\Omega$ is bounded,

(vi) $x_{i} \Omega \in L^{2}\left(\mathrm{R}^{n}, d x\right), i=1, \ldots, n$,

(vii) there exist constants $c>1$ and $\beta$ such that $(c V+\ln \Omega-\beta)^{-} \in$ $L^{p}\left(\mathbf{R}^{n}, d x\right)$ for some $p>2, p>n / 2$.

REMARK. Conditions (vi) and (vii) are decay conditions. The latter can be satisfied, for example, by requiring $c V+\ln \Omega>\beta$ for $|x|>R$ and $V \in$ $L^{P}(\{x$ such that $|x|<R\})$, which is essentially the same as the decay condition in [2].

For $\mu \in \mathscr{T}$, we set $\mathscr{C}=L^{2}\left(\mathbf{R}^{n}, d \mu\right)$ with inner product $\langle\cdot, \cdot\rangle_{d \mu}$ and norm $\|\cdot\|$. We set

$$
h(u, v)=\int_{\mathbf{R}^{n}} \nabla u \cdot \overline{\nabla v} d \mu, \quad H u=-\Delta u-2 \frac{\nabla \Omega \cdot \nabla u}{\Omega}
$$

for $u, v \in \operatorname{Dom}(h)=\operatorname{Dom}(H)=\left\{u \in C^{1}(\mathbf{R})\right.$ such that $D^{a} u$ is bounded for $|\alpha|<1$ and $D^{\alpha} u \in \mathcal{H}$ for $\left.|\alpha|=2\right\}$.

We see from $\$ 2$ that $H$ is an essentially selfadjoint operator which represents the Dirichlet form $h$. By Theorem 3.5, $\bar{H}$ is the generator of an $L^{P}$-contractive semigroup. The additional conditions (v), (vi) and (vii) will insure that $\bar{H}$ generates a hypercontractive semigroup.

THEOREM 5.1. For $u \in \operatorname{Dom}(H)$, there exists $\delta$ such that

$$
\int_{\mathbf{R}^{n}}|u|^{2} \ln |u| d \mu<c \int_{\mathbf{R}^{n}}|\nabla u|^{2} d \mu+\|u\|^{2} \ln \|u\|+\delta\|u\|^{2}
$$

Hence $\bar{H}$ is the generator of a hypercontractive semigroup on the spaces $L^{p}\left(\mathbf{R}^{n}, d \mu\right), 1<p<\infty$.

The proof follows as a sequence of lemmas. 
LeMMA 5.2. For $u \in \operatorname{Dom}(H), \psi=\Omega u, f=\psi e^{x^{2} / 4}=\Omega u e^{x^{2} / 4}$, we have

$$
\begin{aligned}
& (2 \pi)^{-n / 2} \int_{\mathbf{R}^{n}}|f|^{2} \ln |f| e^{-x^{2} / 2} d x<(2 \pi)^{-n / 2} \int_{\mathbf{R}^{n}}|\nabla f|^{2} e^{-x^{2} / 2} d x \\
& +\frac{1}{2}(2 \pi)^{-n / 2} \int_{\mathbf{R}^{n}}|f|^{2} e^{-x^{2} / 2} d x \ln \left((2 \pi)^{-n / 2} \int_{\mathbf{R}^{n}}|f|^{2} e^{-x^{2} / 2} d x\right) .
\end{aligned}
$$

Proof. Equation (8) is Gross' logarithmic Sobolev inequality for Gauss measure (Corollary 4.2 in [4]) which requires only that $f$ have weak gradient in $L^{P}\left(\mathbf{R}^{n},(2 \pi)^{-n / 2} e^{-x^{2} / 2} d x\right)$. Since $f_{x_{i}}=\Omega_{x_{i}} u e^{x^{2} / 4}+\Omega u_{x_{i}} e^{x^{2} / 4}+$ $\Omega u\left(x_{i} / 2\right) e^{x^{2} / 4}$ and $x_{i} \Omega \in L^{2}\left(\mathbf{R}^{n}, d x\right), i=1, \ldots, n$, the lemma follows.

Note that since $|x|^{2} \ln |x|>-1$ for all $x$, we have

$$
\left.\int_{\mathbf{R}^{n}}|| f\right|^{2} \ln |f| \mid e^{-x^{2} / 2} d x<\infty
$$

Since

$$
\left.\left.\int_{\mathbf{R}^{n}}|| \psi\right|^{2} \ln |\psi|\left|d x<\int_{\mathbf{R}^{n}}\right||f|^{2} \ln |f|\left|e^{-x^{2} / 2} d x+\int_{\mathbf{R}^{n}}\right| \psi\right|^{2} \frac{x^{2}}{2} d x
$$

and

$$
\int_{\mathbf{R}^{n}}|\psi|^{2} \frac{x^{2}}{4} d x<\infty
$$

we have also

$$
\left.\int_{\mathbf{R}^{n}}|| \psi\right|^{2} \ln |\psi| \mid d x<\infty
$$

for all $\psi \in \Omega \operatorname{Dom}(H)$. In particular, for $\psi=\Omega$ we have

$$
\int_{\mathbf{R}^{n}}\left|\Omega^{2} \ln \Omega\right| d x<\infty
$$

LEMMA 5.3. There exists $\gamma_{1}$ such that for $\psi=\Omega u, u \in \operatorname{Dom}(H)$,

$$
\begin{aligned}
\int_{\mathbf{R}^{n}}|\psi|^{2} \ln |\psi| d x< & \int_{\mathbf{R}^{n}}|\nabla \psi|^{2} d x+\frac{1}{2} \int_{\mathbf{R}^{n}}|\psi|^{2} d x \ln \left(\int_{\mathbf{R}^{n}}|\psi|^{2} d x\right) \\
& +\gamma_{1} \int_{\mathbf{R}^{n}}|\psi|^{2} d x .
\end{aligned}
$$

Proof. We have

$$
\begin{aligned}
\int_{\mathbf{R}^{n}} & \left|\nabla\left(\psi e^{x^{2} / 4}\right)\right|^{2} e^{-x^{2} / 2} d x \\
& =\int_{\mathbf{R}^{n}}|\nabla \psi|^{2} d x+\int_{\mathbf{R}^{n}} \frac{x}{2} \cdot \nabla\left(|\psi|^{2}\right) d x+\int_{\mathbf{R}^{n}}|\psi|^{2} \frac{x^{2}}{4} d x \\
& =\int_{\mathbf{R}^{n}}|\nabla \psi|^{2} d x-\frac{n}{2} \int_{\mathbf{R}^{n}}|\psi|^{2} d x+\int_{\mathbf{R}^{n}}|\psi|^{2} \frac{x^{2}}{4} d x
\end{aligned}
$$


noting that for $i=1, \ldots, n$, the equality

$$
\int_{\mathbf{R}^{n}} x_{i}\left(|\psi|^{2}\right)_{x_{i}} d x=-\int_{\mathbf{R}^{n}}|\psi|^{2} d x
$$

is a straightforward integration by parts calculation similar to the one described in $\$ 2$. Hence substituting $f=\psi e^{x^{2} / 4}$ into (8), we obtain (9) with $\gamma_{1}=(n / 2)\left(1+\frac{1}{2} \ln 2 \pi\right)$.

LEMMA 5.4. There exists $\gamma_{2}$ such that for all $\psi \in \Omega \operatorname{Dom}(H)$,

$$
\int_{\mathbf{R}^{n}}\left[(c-1)|\nabla \psi|^{2}+\left(c V+\gamma_{2}+\ln \Omega\right)|\psi|^{2}\right] d x>0 .
$$

Proof. We set

$$
W=(c V+\ln \Omega-\beta) /(c-1) .
$$

Proposition 6.4 in [3] states that if $f \in L^{p}\left(\mathbf{R}^{n}, d x\right)$ for some $p>2, p>n / 2$, then the form associated with $f$,

$$
t_{1}(\psi, \varphi)=\int_{\mathbf{R}^{n}} \psi \psi \bar{\varphi} d x
$$

is a relatively small perturbation of the Dirichlet form $t$, associated with the selfadjoint operator $-\Delta$ on $L^{2}\left(\mathbf{R}^{n}, d x\right)$. Theorem VI-(1.33) in [5] implies that if a form $t$ is bounded from below and if a symmetric form $t_{1}$ is a relatively small pertubation of $t$, then the form $t+t_{1}$ is bounded from below. Thus, setting $f=W^{-}$, we have $t+t_{1}$ bounded from below, by, say, $a$.

We know that $\Omega \operatorname{Dom}(H)$ is contained in $\operatorname{Dom}(t)$ since $\psi \in \Omega \operatorname{Dom}(H)$ has weak first derivatives in $L^{2}\left(\mathbf{R}^{n}, d x\right)$. In addition, for $\psi=\Omega u, u \in$ $\operatorname{Dom}(H)$,

$$
\begin{aligned}
\int_{\mathbf{R}^{n}}|V||\psi|^{2} d x & =\int_{\mathbf{R}^{n}}|u|^{2}\left|\frac{\Delta \Omega}{\Omega}\right| \Omega^{2} d x<\infty, \\
\left.\int_{\mathbf{R}^{n}}|| \psi\right|^{2} \ln \Omega \mid d x & =\int_{\mathbf{R}^{n}}|u|^{2}\left|\Omega^{2} \ln \Omega\right| d x<\infty
\end{aligned}
$$

so that $\Omega \operatorname{Dom}(H) \subset \operatorname{Dom}\left(t_{1}\right)$. Hence

$$
\int_{\mathbf{R}^{n}}\left(|\nabla \psi|^{2}+W|\psi|^{2}\right) d x>\left(t+t_{1}\right)(\psi)+\int_{\mathbf{R}^{n}} W^{+}|\psi|^{2} d x>a \int_{\mathbf{R}^{n}}|\psi|^{2} d x
$$

and the lemma follows with $\gamma_{2}=-\beta-(c-1) a$.

Proof OF Theorem 5.1. We can integrate by parts to obtain

$$
\int_{\mathbf{R}^{n}} \nabla \psi \cdot \bar{\nabla} \varphi d x=-\langle\Delta \psi, \varphi\rangle_{d x}
$$

for all $\varphi, \psi \in \Omega \operatorname{Dom}(H)$. It should be noted that this integration by parts does not necessarily follow with weaker smoothness conditions on $\Omega$. 
Now we have for $u \in \operatorname{Dom}(H), \psi=\Omega u$,

$$
\int_{\mathbf{R}^{n}}|\nabla u|^{2} d \mu=\langle H u, u\rangle_{d \mu}=\langle S \psi, \psi\rangle_{d x}=\int_{\mathbf{R}^{n}}\left(|\nabla \psi|^{2}+V|\psi|^{2}\right) d x .
$$

Hence adding (9) and (10), we obtain

$$
\begin{aligned}
& c \int_{\mathbf{R}^{n}}|\nabla u|^{2} d \mu=c \int_{\mathbf{R}^{n}}\left(|\nabla \psi|^{2}+V|\psi|^{2}\right) d x \\
&> \int_{\mathbf{R}^{n}}|\psi|^{2} \ln |\psi| d x-\frac{1}{2} \int_{\mathbf{R}^{n}}|\psi|^{2} d x \ln \left(\int_{\mathbf{R}^{n}}|\psi|^{2} d x\right) \\
& \quad+\int_{\mathbf{R}^{n}}\left(-\gamma_{1}-\gamma_{2}-\ln \Omega\right)|\psi|^{2} d x \\
&= \int_{\mathbf{R}^{n}}|u|^{2} \ln |u| d \mu+\int_{\mathbf{R}^{n}}|u|^{2}(\ln \Omega) d \mu-\frac{1}{2} \int_{\mathbf{R}^{n}}|u|^{2} d \mu \ln \left(\int_{\mathbf{R}^{n}}|u|^{2} d \mu\right) \\
& \quad-\left(\gamma_{1}+\gamma_{2}\right) \int_{\mathbf{R}^{n}}|u|^{2} d \mu-\int_{\mathbf{R}^{n}}|u|^{2} \ln \Omega d \mu .
\end{aligned}
$$

We note that all terms are finite. The inequality ( 7$)$ follows with $\delta=-\left(\gamma_{1}\right.$ $\left.+\gamma_{2}\right)$. That $\bar{H}$ generates a hypercontractive semigroup follows from Theorem 4.5. Hence the proof of Theorem 5.1 is complete.

REMARK. It can be shown that for measures $d \mu=\Omega^{2} d x$ on $\mathbf{R}^{1}$, where $\Omega$ satisfies essentially the same decay conditions described in this section but where $\Omega^{\prime}$ has a simple jump discontinuity, the associated selfadjoint operator generates a hypercontractive semigroup. The only major difference in the proof is that the operator inequality in Lemma 3.4 has to be replaced by a form inequality which accounts for the discontinuity.

Also, measures can be exhibited on $R^{1}$ with support $[0,1]$ such that the associated operator generates a hypercontractive semigroup. The decay conditions are replaced by boundary conditions on $\Omega$ at 0 and 1 . An example is the measure $d \mu=\Omega^{2} d x$ where $\Omega(x)=(30)^{1 / 2} x(1-x)$ on $[0,1], \Omega(x)=0$ otherwise.

REMARK. By placing more restrictive decay conditions on measures similar to Eckmann's, J. Rosen [10] obtained a class of measures for which the corresponding semigroup of operators is supercontractive, i.e. for $1<p$, $q<\infty,\left\|e^{-t \bar{H}}\right\|_{p, q}<\infty$ for all $t>0$.

It seems reasonable to assume that by similarly restricting the measures discussed in this paper, while retaining the weak differentiability conditions, we could obtain a class of supercontractive semigroups.

We conclude with an example of a measure on $\mathbf{R}^{1}$ such that the associated operator generates an $L^{P}$-contractive semigroup which fails to be hypercontractive.

We remarked at the end of $\$ 2$ that the operator $H$ associated with the 
Dirichlet form for the measure $d \mu=e^{-2|x|} d x$ is essentially selfadjoint with $\operatorname{Dom}(H)=\left\{u \in C^{1}(\mathbf{R})\right.$ such that $u, u^{\prime}$ are bounded, $\left.u^{\prime \prime} \in \mathcal{C}=L^{2}(\mathbf{R}, d \mu)\right\}$. By Theorem 3.5, $\bar{H}$ generates an $L^{p}$-contractive semigroup. As mentioned in the proof of Corollary 4.6 , if $\bar{H}$ generates a hypercontractive semigroup, then $\bar{H}$ is a Sobolev generator on $[1, \infty)$. In particular, there exist constants $c>0$ and $\delta$ such that for $u \in \operatorname{Dom}(H)$,

$$
c\langle H u, u\rangle \geqslant \int_{\mathbf{R}}|u|^{2} \ln |u| d \mu-\frac{1}{2} \int_{\mathbf{R}}|u|^{2} d \mu \ln \left(\int_{\mathbf{R}}|u|^{2} d \mu\right)+\delta \int_{\mathbf{R}}|u|^{2} d \mu .
$$

For $\psi \in \Omega \operatorname{Dom}(H)$ with compact support away from the origin, this can be written

$$
\begin{gathered}
c \int_{\mathbf{R}}\left(\left|\psi^{\prime}\right|^{2}+V|\psi|^{2}\right) d x>\int_{\mathbf{R}}|\psi|^{2} \ln |\psi| d x-\int_{\mathbf{R}}|\psi|^{2} \ln \Omega d x \\
-\frac{1}{2} \int_{\mathbf{R}}|\psi|^{2} d x \ln \left(\int_{\mathbf{R}}|\psi|^{2} d x\right)+\delta \int_{\mathbf{R}}|\psi|^{2} d x .
\end{gathered}
$$

We fix a nonzero $\psi \in C_{0}^{\infty}(0,1)$ and define $\psi_{n}(x)=\psi(x-n)$. Recalling that $V=1$, we see that as $n \rightarrow \infty$, all terms of (11) remain fixed except

$$
-\int_{\mathbf{R}}\left|\psi_{n}\right|^{2} \ln \Omega d x=\int_{n}^{n+1}\left|\psi_{n}\right|^{2} x d x \rightarrow \infty \quad \text { as } n \rightarrow \infty .
$$

Hence (11) cannot hold and $\bar{H}$ does not generate a hypercontractive semigroup. Note that $\Omega=e^{-|x|}$ fails to satisfy condition (vii) of this section.

\section{REFERENCES}

1. A. Beurling and J. Deny, Espaces de Dirichlet. I. Le cas élémentaire, Acta Math. 99 (1958), 203-224.

2. J.-P. Eckmann, Hypercontractivity for anharmonic ascillators, J. Functional Analysis 16 (1974), 388-404.

3. W. Faris, Quadratic forms and essential self-adjointness, Helv. Phys. Acta 45 (1972), 1074-1088.

4. L. Gross, Logarithmic Soboleo inequalities, Amer. J. Math. 97 (1975), 1061-1083.

5. T. Kato, Perturbation theory for linear operators, Springer-Verlag, New York, 1966.

6. __ Schrodinger operators with singuiar potentials, Israel J. Math. 13 (1973), 135-148.

7. E. Nelson, The free Markoo field, J. Functional Analysis 12 (1973), 211-227.

8. M. Reed and B. Simon, Methods of modern mathematical physics. II. Fourier analysis, self-adjointness, Academic Press, New York, 1975.

9. , Methods of modern mathematical physics. IV. Analysis of operators, Academic Press, New York, 1978.

10. J. Rosen, Sobolev inequalities for weight spaces and supercontractioity, Trans. Amer. Math. Soc. 222 (1976), 367-376.

11. I. E. Segal, Construction of non-linear local quantum processes. I, Ann. of Math. (2) 92 (1970), 462-481; II, Invent. Math. 14 (1971), 211-241.

Department of Mathematics, Loustana State Universtry, Baton Rouge, loutsina 70803 